\title{
Terapias alternativas utilizadas em pacientes oncológicos em cuidados paliativos: revisão integrativa
}

\author{
Alternative therapies used in oncological patients in paliaival care: integrative review \\ Terapias alternativas utilizadas en pacientes oncológicos en cuidados paliativos: revisión \\ integrativa
}

Elisângela Faustino Farias da Costa ${ }^{1 *}$, Silvia Cristina Fürbringer e Silva ${ }^{2}$, Michelle Ribeiro da Silva ${ }^{3}$, Gedielma Maria da Silva Martins ${ }^{4}$, Juliana Beltrão Mulatinho ${ }^{5}$.

\begin{abstract}
RESUMO
Objetivo: Identificar os tipos de TC mais abordadas na literatura em CP de pacientes oncológicos. Métodos: Para o desenvolvimento da presente revisão foram percorridas as seguintes etapas: escolha dos artigos; avaliação dos estudos incluídos; interpretações dos resultados, e apresentação da revisão integrativa. Foi realizada uma revisão integrativa da literatura com artigos publicados entre os anos 2016 e 2020 . O estudo selecionou 11 artigos, e os resultados encontrados indicam que a publicação sobre o tema tem aumentado nos últimos anos, com muitos resultados clínicos positivos, mas que ainda são necessários mais estudos clínicos, incluindo grupos controles. Resultados: Esse estudo não encontrou nenhuma terapia que tratasse do tema de terapias complementares, através das bases de dados utilizadas, que foram escolhidas justamente por serem as mais abrangentes. Isso evidencia a necessidade do desenvolvimento de pesquisas clínicas baseadas em evidências. Considerações finais: Apesar de haver algumas publicações nacionais sobre CP em pacientes oncológicos, esse estudo não encontrou nenhuma que tratasse do tema de terapias complementares, através das bases de dados utilizadas, que foram escolhidas justamente por serem as mais abrangentes.
\end{abstract}

Palavras-chave: Terapias complementares, Cuidados paliativos, Neoplasias.

\begin{abstract}
Objective: To identify the types of CT most frequently addressed in the literature on PC of cancer patients. Methods: For the development of this review, the following steps were taken: choice of articles; evaluation of the included studies; interpretations of the results, and presentation of the integrative review. An integrative literature review was carried out with articles published between the years 2016 and 2020. The study selected 11 articles, and the results found indicate that the publication on the subject has increased in recent years, with many positive clinical results, but which are still Further clinical studies are needed, including control groups. Results: This study did not find any therapy that addressed the topic of complementary therapies, through the databases used, which were chosen precisely because they are the most comprehensive. This highlights the need for the development of evidence-based clinical research. Final considerations: Although there are some national publications on PC in cancer patients, this study found none that dealt with the topic of complementary therapies, through the databases used, which were chosen precisely because they are the most comprehensive.
\end{abstract}

Key words: Complementary therapies, Palliative care, Neoplasms.

\section{RESUMEN}

Objetivo: Identificar los tipos de TC más abordados en la literatura sobre CP de pacientes oncológicos. Métodos: Para el desarrollo de esta revisión se siguieron los siguientes pasos: elección de artículos;

\footnotetext{
1 Universidade Brasileira (IBRATI), São Caetano do Sul - SP. *E-mail: elisangela-farias28@hotmail.com

2 Universidade de São Paulo (SOPATI), Bela vista - SP.

3 Universidade Federal de Pernambuco (UFPE), Recife - PE.

${ }^{4}$ Universidade Salgado de Oliveira (UNIVERSO), Recife - PE.

${ }^{5}$ Fundação Osvaldo Cruz (FIOCRUS), Rio de Janeiro - RJ.
}

SUBMETIDO EM: 3/2021

ACEITO EM: 4/2021

PUBLICADO EM: 5/2021 
evaluación de los estudios incluidos; interpretaciones de los resultados y presentación de la revisión integradora. Se realizó una revisión integradora de la literatura con artículos publicados entre los años 2016 y 2020. El estudio seleccionó 11 artículos, y los resultados encontrados indican que la publicación sobre el tema ha aumentado en los últimos años, con muchos resultados clínicos positivos, pero que aún son Se necesitan más estudios clínicos, incluidos los grupos de control. Resultados: Este estudio no encontró ninguna terapia que abordara el tema de las terapias complementarias, a través de las bases de datos utilizadas, las cuales fueron elegidas precisamente por ser las más completas. Esto resalta la necesidad de desarrollar investigación clínica basada en evidencia. Consideraciones finales: Si bien existen algunas publicaciones nacionales sobre $\mathrm{CP}$ en pacientes con cáncer, este estudio no encontró ninguna que abordara el tema de las terapias complementarias, a través de las bases de datos utilizadas, las cuales fueron elegidas precisamente por ser las más completas.

Palabras clave: Terapias complementarias, Cuidados paliativos, Neoplasias.

\section{INTRODUÇÃO}

De acordo com a Organização Mundial da Saúde (OMS), os cuidados paliativos melhoram a qualidade de vida dos pacientes e, também, de suas famílias, que enfrentam problemas associados a doenças potencialmente fatais, sejam elas físicas, psicossociais ou espirituais. Afinal, abordar o sofrimento envolve cuidar de questões além dos sintomas físicos. Assim, estes cuidados utilizam uma abordagem em equipe para apoiar os pacientes e seus cuidadores. Isso inclui atender às necessidades práticas e fornecer aconselhamento de luto. Nesse caso, os profissionais em CP buscam oferecer um sistema de apoio para ajudar os pacientes a viver o mais ativamente possível até sua morte (WORLD HEALTH ORGANIZATION (WHO, 2018).

Dentro desse contexto, as Terapias Complementares (TC) surgem como sistemas médicos e recursos terapêuticos complexos empregados para promoção do amplo cuidado em saúde, valorizando a autonomia, cultura e o ambiente dos indivíduos. Dessa forma, como resultado da mudança de paradigmas relacionados à assistência em saúde, da demanda popular, de recomendações da Organização Mundial de Saúde (OMS) e de diretrizes nacionais relacionadas ao tema, foi aprovada, em 2006, a Política Nacional de Práticas Integrativas e Complementares no Sistema Único de Saúde SUS (PNPIC) (BRASIL, 2015).

A PNPIC veio atender, principalmente, a crescente necessidade de se ampliar o conhecimento sobre algumas experiências em TC que já vinham sendo desenvolvidas na rede pública de muitos municípios e estados, além de apoiar essas terapias, incorporá-las e implementá-las ao SUS. Entre essas TC se destacam as que se encontram no âmbito da medicina tradicional chinesa (como a acupuntura), além da homeopatia, fitoterapia, medicina antroposófica e do termalismo/crenoterapia (BRASIL, 2015).

De acordo com Shin N e Park J (2018), existem vários tratamentos paliativos que podem diminuir os efeitos colaterais dos medicamentos contra o câncer ou melhorar a qualidade de vida desses pacientes de outras formas. Por exemplo, a acupuntura, a acupressão e a hipnoterapia podem aliviar dores, náuseas e vômitos, a aromaterapia e a massagem terapêutica podem trazer maior bem-estar e a musicoterapia pode reduzir distúrbios do humor. Entretanto, o mesmo autor alerta para o fato de que as TC devem ser apresentadas aos pacientes não como uma forma de cura para seus males, e sim como um paliativo capaz de ajudá-los a melhorar sua qualidade de vida.

Câncer é um termo genérico, utilizado para denominar um grupo de mais de 100 doenças distintas, que possuem causas multifatoriais e têm em comum o crescimento desordenado de células que, por sua vez, invadem tecidos e órgãos. Ao se dividirem com rapidez, estas células podem se tornar muito agressivas e incontroláveis, levando a formação de massas tumorais, que podem sofrer metástases e se espalharem para outras regiões do corpo (INSTITUTO NACIONAL DO CÂNCER (INCA), 2018).

Assim, a diminuição do sofrimento está diretamente relacionada com o controle do sofrimento álgico e com a promoção de conforto físico, psíquico e até mesmo espiritual aos pacientes assistidos. Apesar de haver algum conhecimento acerca das necessidades dos pacientes oncológicos, na prática o tratamento do câncer 
é muito limitado. Por esse motivo, é necessário entender melhor as necessidades do paciente terminal em câncer, e correlacionar ao que está sendo aplicado no tratamento da doença, a fim de proporcionar-Ihes uma melhor condição até o óbito.

Diante desse quadro, o objetivo deste trabalho foi fazer uma nova abordagem de tratamento para pacientes oncológicos, que se disponha a fornecer aos pacientes bem-estar e melhor qualidade de vida, o que pode ser realizado através dos Cuidados Paliativos $(\mathrm{CP})$, que é uma modalidade de tratamento que dedica a atenção a pacientes com doenças crônicas e/ou degenerativas, e a vida de pacientes em fase terminal.

\section{MÉTODOS}

Trata-se de uma revisão integrativa da literatura, considerada um método de estudo que possibilita a síntese do conhecimento de um determinado assunto, além de apontar lacunas, que precisam ser preenchidas. Foi escolhido este método porque este tipo de revisão consiste na construção de uma análise ampla da literatura, permitindo a inclusão de múltiplos estudos com diferentes delineamentos de pesquisas, contribuindo para discussões sobre seus métodos e resultados, assim como reflexões sobre a realização de futuros estudos. Portanto, o propósito inicial deste método de pesquisa é obter um profundo entendimento de um determinado fenômeno, baseando-se em estudos anteriores (RADOSSI AL, 2017).

Para o desenvolvimento da presente revisão foram percorridas as seguintes etapas: escolha dos artigos; avaliação dos estudos incluídos; interpretações dos resultados, e apresentação da revisão integrativa. Para orientar a pesquisa, elaborou-se a seguinte pergunta: Quais são as terapias complementares abordadas na literatura para tratamento paliativo em pacientes oncológicos? O problema do estudo se desenvolve no sentido de identificar de que forma as Terapias Complementares (TC) influenciam no processo dos CP em pacientes oncológicos.

A coleta de dados ocorreu durante o mês de maio de 2019 e foram selecionados artigos das bases científicas: PubMed e Biblioteca Virtual em Saúde/Medline (que inclui as bases de dados Scientific Electronic Library (SciELO) e Literatura Latino-Americana e do Caribe em Ciências da Saúde (Lilacs)). Foram utilizados descritores presentes nos descritores em ciências da saúde (DECS): Terapias complementares (Complementary therapies); Cuidados paliativos (Palliative care); Neoplasias (Neoplasms). Foi utilizado, também, o operador booleano AND.

Como critérios de inclusão foram utilizados os seguintes: artigos publicados entre os anos 2014 e 2018; disponíveis na íntegra online; escritos nos idiomas português e inglês, cujo conteúdo fosse sobre estudos clínicos, relatos de casos, meta-análises, revisão sistemática ou integrativa.

Foram excluídos os artigos que não se encaixassem nos critérios descritos, bem como aqueles que, após a leitura de seus títulos, resumos ou leitura na íntegra não se apresentassem com conteúdo relevante para esse estudo. Também foram descartados os artigos repetidos nas plataformas de busca (que foram utilizados apenas uma vez).

\section{RESULTADOS}

Trata-se de uma pesquisa bibliográfica para a resolução da hipótese por meio de referenciais teóricos publicados, analisando e discutindo as várias contribuições científicas. Esse tipo de pesquisa trará subsídios para o conhecimento sobre o que foi pesquisado, como e sob que enfoque e/ou perspectivas foi tratado 0 assunto apresentado na literatura científica.

Como procedimentos técnicos, foram utilizadas pesquisas bibliográficas, em alguns artigos científicos e este estudo foi caracterizado pela revisão de literatura nacional, nas bases de dados eletrônicas SciELO, o banco de dados da LILACS e PubMed. Onde foram encontrados 121 artigos, após a aplicação das seguintes palavras chaves na pesquisa: Terapias Complementares, Cuidados Paliativos, Neoplasias. Por meio da leitura dos artigos, foram selecionados 13 artigos. Após a leitura dos mesmos, foram escolhidos 11 artigos, que se encontram descritos no Quadro 1, através das variáveis: autor(es), base de dados, periódicos e ano de publicação. 
Quadro 1 - Relação dos estudos incluídos na revisão integrativa.

\begin{tabular}{|c|c|c|c|}
\hline Autor(es) e Título & $\begin{array}{l}\text { Periódico e Ano de } \\
\text { Publicacão }\end{array}$ & Objetivo & Conclusão \\
\hline $\begin{array}{l}\text { Axtner } J \text {, et al. Health services } \\
\text { research of integrative oncology in } \\
\text { palliative care of patients with } \\
\text { advanced pancreatic câncer. }\end{array}$ & BMC Cancer (2016) & 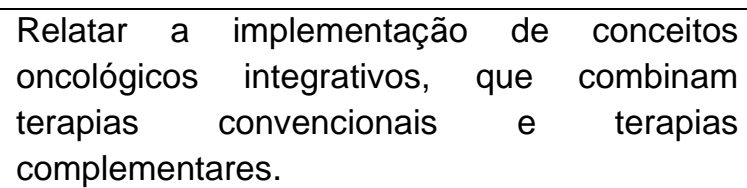 & $\begin{array}{l}\text { O tratamento de TC pode ser implementado com } \\
\text { sucesso no cuidado diário de pacientes que } \\
\text { sofrem de câncer pancreático avançado. }\end{array}$ \\
\hline $\begin{array}{l}\text { Ben } A E \text {, et al. Integrating family } \\
\text { medicine and complementary } \\
\text { medicine in cancer care: A cross- } \\
\text { cultural perspective. }\end{array}$ & $\begin{array}{l}\text { Patient Education and } \\
\text { Counseling (2014) }\end{array}$ & $\begin{array}{l}\text { Descrever o estudo de caso de uma paciente } \\
\text { que recebeu tratamento paliativo para câncer } \\
\text { de mama avançado. }\end{array}$ & $\begin{array}{l}\text { A TC é ideal para os cuidados de suporte holísticos } \\
\text { centrados no paciente. A inclusão de profissionais } \\
\text { de TC treinados em uma equipe integrativa } \\
\text { multidisciplinar pode melhorar a perspectiva } \\
\text { biopsico-social-espiritual. }\end{array}$ \\
\hline $\begin{array}{l}\text { Ben } \mathrm{AE} \text {, et al. Integrative palliative } \\
\text { care: complementary medicine in } \\
\text { oncology. }\end{array}$ & $\begin{array}{l}\text { Clinical Journal of } \\
\text { Oncology Nursing } \\
(2017)\end{array}$ & $\begin{array}{l}\text { Apresentar o esforço colaborativo de dois } \\
\text { profissionais de medicina complementar e } \\
\text { integrativa (CIM) que cuidam de três pacientes } \\
\text { com câncer. }\end{array}$ & $\begin{array}{l}\text { O efeito da abordagem combinada foi sinérgico, } \\
\text { capacitando os pacientes e os profissionais da } \\
\text { CIM, reduzindo a carga de sintomas e melhorando } \\
\text { a qualidade de vida e a função. }\end{array}$ \\
\hline $\begin{array}{l}\text { Chaoul } A \text {, et al. Randomized trial } \\
\text { of Tibetan yoga in patients with } \\
\text { breast cancer undergoing } \\
\text { chemotherapy. }\end{array}$ & Cancer (2018) & $\begin{array}{l}\text { Examinar os efeitos de um programa de yoga } \\
\text { tibetana (TYP) versus um programa de } \\
\text { alongamento (STP) e cuidados habituais (UC). }\end{array}$ & $\begin{array}{l}\text { Participar do TYP durante a quimioterapia resultou } \\
\text { em benefícios de curto prazo modestos na } \\
\text { qualidade do sono, com benefícios a longo prazo. }\end{array}$ \\
\hline $\begin{array}{l}\text { Chung VC, et al. Chinese herbal } \\
\text { medicine for symptom } \\
\text { management in cancer palliative } \\
\text { care: systematic review and meta- } \\
\text { analysis. }\end{array}$ & Medicine (2016) & $\begin{array}{l}\text { Conduzir uma revisão sistemática com meta- } \\
\text { análise para resumir os resultados de ensaios } \\
\text { clínicos controlados randomizados sobre o uso } \\
\text { de fitoterápicos chineses ( } \mathrm{CHM}) \text {. }\end{array}$ & $\begin{array}{l}\text { A CHM pode ser considerada como um } \\
\text { complemento aos cuidados convencionais no } \\
\text { tratamento da dor em pacientes com câncer, } \\
\text { também pode ser considerada uma alternativa aos } \\
\text { cuidados convencionais. }\end{array}$ \\
\hline $\begin{array}{l}\text { Grégoire C, et al. Group } \\
\text { interventions to reduce emotional } \\
\text { distress and fatigue in breast } \\
\text { cancer patients: a 9-month follow- } \\
\text { up pragmatic trial. }\end{array}$ & $\begin{array}{l}\text { British Journal } \\
\text { Cancer (2017) }\end{array}$ & $\begin{array}{l}\text { Avaliar a eficácia de } 3 \text { intervenções em grupo, } \\
\text { com base na terapia cognitivo-comportamental } \\
\text { (TCC), yoga e auto-hipnose, }\end{array}$ & $\begin{array}{l}\text { Os resultados mostraram que as intervenções } \\
\text { mente-corpo parecem ser uma abordagem } \\
\text { interessante para melhorar o bem-estar das } \\
\text { pacientes com câncer de mama. }\end{array}$ \\
\hline
\end{tabular}




\begin{tabular}{|c|c|c|c|}
\hline Autor(es) e Título & $\begin{array}{l}\text { Periódico e Ano de } \\
\text { Publicacão }\end{array}$ & Objetivo & Conclusão \\
\hline $\begin{array}{l}\text { Lau } \mathrm{CH} \text {, et al. Acupuncture and } \\
\text { related therapies for symptom } \\
\text { management in palliative cancer } \\
\text { care: systematic review and meta- } \\
\text { analysis. }\end{array}$ & Medicine (2016) & $\begin{array}{l}\text { Realizar uma revisão sistemática e meta- } \\
\text { análise para resumir as melhores evidências } \\
\text { atuais sobre a acupuntura e terapias } \\
\text { relacionadas para o tratamento do câncer } \\
\text { paliativo. }\end{array}$ & $\begin{array}{l}\text { A acupuntura e as terapias relacionadas são } \\
\text { eficazes na redução da dor, fadiga e na melhoria } \\
\text { da qualidade de vida quando comparadas à } \\
\text { intervenção convencional. }\end{array}$ \\
\hline $\begin{array}{l}\mathrm{Ng} \mathrm{CG} \text {, et al. The effect of } 5 \\
\text { minutes of mindful breathing to the } \\
\text { perception of distress and } \\
\text { physiological responses in } \\
\text { palliative care cancer patients: a } \\
\text { randomized controlled study. }\end{array}$ & $\begin{array}{l}\text { Journal of Palliative } \\
\text { Medicine (2016) }\end{array}$ & $\begin{array}{l}\text { Estudar a eficácia de } 5 \text { minutos de respiração } \\
\text { consciente (MB) para redução rápida de } \\
\text { sofrimento em um ambiente paliativo. }\end{array}$ & $\begin{array}{l}\text { Houve redução significativa do sofrimento } \\
\text { percebido, da pressão arterial, da pulsação, da } \\
\text { frequência respiratória e da resposta galvânica da } \\
\text { pele. }\end{array}$ \\
\hline $\begin{array}{l}\text { Radossi AL, et al. A systematic } \\
\text { review of integrative clinical trials } \\
\text { for supportive care in pediatric } \\
\text { oncology: a report from the } \\
\text { International Society of Pediatric } \\
\text { Oncology, T\&CM collaborative. }\end{array}$ & $\begin{array}{l}\text { Supportive Care in } \\
\text { Cancer }(2017)\end{array}$ & $\begin{array}{l}\text { Realizar uma revisão sistemática com o } \\
\text { objetivo de avaliar as evidências de TC para } \\
\text { uma variedade de indicações de cuidados de } \\
\text { apoio entre as crianças com câncer. }\end{array}$ & $\begin{array}{l}\text { A pesquisa revelou numerosos estudos clínicos } \\
\text { investigando o uso de TC para fins de cuidados de } \\
\text { suporte em oncologia pediátrica em países de alta } \\
\text { renda, alta renda média, e baixa renda média. }\end{array}$ \\
\hline $\begin{array}{l}\text { Shalom SI, et al. Impact of a } \\
\text { complementary/integrative } \\
\text { medicine program on the need for } \\
\text { supportive cancer care-related } \\
\text { medications. }\end{array}$ & $\begin{array}{l}\text { Supportive Care in } \\
\text { Cancer }(2017)\end{array}$ & $\begin{array}{l}\text { Examinar o impacto das TC na necessidade de } \\
\text { medicamentos de suporte relacionados ao } \\
\text { tratamento do câncer. }\end{array}$ & $\begin{array}{l}\text { Os resultados evidenciaram que os tratamentos } \\
\text { com TC podem reduzir a necessidade de } \\
\text { analgésicos não opioides em pacientes com } \\
\text { câncer de mama ou ginecológico. }\end{array}$ \\
\hline $\begin{array}{l}\text { Wu X, et al. Effectiveness of } \\
\text { acupuncture and related therapies } \\
\text { for palliative care of cancer: } \\
\text { overview of systematic reviews. }\end{array}$ & $\begin{array}{l}\text { Scientific } \\
(2015)\end{array}$ & $\begin{array}{l}\text { Resumir as evidências sobre a acupuntura para } \\
\text { cuidados paliativos de câncer através de uma } \\
\text { revisão sistemática. }\end{array}$ & $\begin{array}{l}\text { A acupuntura parece ser relativamente segura, e } \\
\text { pode ser considerada como uma forma } \\
\text { complementar de cuidados paliativos para o } \\
\text { câncer. }\end{array}$ \\
\hline
\end{tabular}

Fonte: Costa EFF, et al., 2016 


\section{DISCUSSÃO}

Os estudos selecionados seguem nesse tópico com a análise de seus conteúdos, sendo criticamente discutidos e comparados. Os dados do Quadro 1, referente aos 11 artigos selecionados, de maior relevância e com conteúdo mais direcionado para as TC encontradas, a grande maioria se encontrava no intervalo de tempo dos últimos cinco anos.

Conrad AC, et al. (2014), realizou um estudo na Alemanha, e os pesquisadores afirmam que também em seu país, assim como no Reino Unido, uma alta proporção de pacientes com câncer faz uso das TC, sendo que cerca de $40 \%$ a $50 \%$ de todos os pacientes a usam e, em algumas entidades, como câncer de mama, até $90 \%$ foi relatado. Assim, os autores realizaram uma pesquisa sobre profissionais em CP em relação às atitudes em relação as TC. Foi feita uma pesquisa por meio de um questionário padronizado enviado a todos os membros da Sociedade Alemã de CP. Um total de 690 questionários (19\%) foram devolvidos (49\% médicos, 35\% enfermeiros, 3\% psicólogos). Conforme os resultados, concluíram que a aceitação das TC é alta (92\% para TC e 54\% para medicina alternativa). A maioria dos participantes já havia sido questionada sobre CAM por pacientes (95\%) e parentes (89\%). Entre esses, $44 \%$ já utilizaram métodos complementares e $5 \%$ de métodos alternativos.

Apesar de não trazer resultados clínicos, foi incluído nessa revisão integrativa por sua importância em demonstrar que em outros países, essas TC estão sendo reconhecidas e valorizadas por profissionais de saúde como importantes auxiliares para o cuidado em saúde de seus pacientes. Segundo os resultados do estudo, entre os TC mais recomendados por médicos estava as técnicas de relaxamento (91\%), terapia com exercícios $(84 \%)$, cuidados espirituais e oração $(82 \%)$, musicoterapia $(80 \%)$ e arteterapia $(79 \%)$. Entre os enfermeiros, as recomendações positivas foram mais frequentes, onde a estimulação basal é recomendada por $89 \%$, seguida pelas massagens (88\%), aromaterapia (79\%) e homeopatia (63\%) (CONRAD AC, et al., 2014).

Segundo Ben A, et al. (2014), trazem um relato de caso de uma paciente árabe de 27 anos que recebeu tratamento paliativo para câncer de mama avançado, sendo encaminhada para uma consulta de medicina complementar, em um departamento convencional de oncologia em Israel. O estudo traz o resultado do impacto da equipe integrativa de profissionais de TC, que incluía três médicos de família treinados em TC e um praticante de medicina chinesa, preocupados com o bem-estar da paciente e especificamente no alívio de suas debilitantes ondas de calor e insônia, além da ansiedade, dispneia, dor, fadiga e náuseas. Foram utilizadas a acupuntura e acupressão, fitoterapia tradicional islâmica (suplemento à base de ervas Cimicifuga), imaginação guiadas (incluindo para uso em auto experiência em casa) e foi recomendado que a paciente escrevesse um diário com seus pensamentos e medos. Foi confirmada a melhora na qualidade de vida e dos sintomas por meio dos resultados comparativos entre os questionários Edmonton Symptom Assessment Scale (ESAS) e Measure Yourself Concerns and Well-Being (MYCAW) administrados nas sessões de avaliação inicial e de acompanhamento.

O estudo de Ben A, et al. (2014), foi importante porque durante o tratamento foram feitas várias avaliações e adaptações do tratamento com TC, até se estabelecer o protocolo que se mostrou ideal para a paciente, produzindo resultados positivos. O estudo concluiu que as TC foram eficazes e que a inclusão de profissionais de TC treinados em uma equipe integrativa multidisciplinar pode melhorar a perspectiva biopsico-socialespiritual dos pacientes e fornecer terapias práticas adicionais que melhorem a qualidade de vida dos que enfrentam o câncer. Os pesquisadores afirmam, e esse estudo concorda, que a TC é ideal para os cuidados de suporte holísticos centrados no paciente., e que a inclusão de profissionais de TC treinados, em uma equipe integrativa multidisciplinar, pode melhorar a perspectiva biopsico-social-espiritual e fornecer terapias práticas adicionais que melhorem a qualidade de vida dos pacientes que enfrentam o câncer.

No caso desse estudo, sua importância foi a de demonstrar que a integração desses profissionais em TC em uma equipe de CP levou, para os demais profissionais e pacientes, um paradigma diferente de cuidado, onde foi possível combinar seu conhecimento e experiência, ao mesmo tempo em que forneceu uma perspectiva única sobre as preocupações dos pacientes que precisam ser abordadas, como em um dos casos, que houve a necessidade de toque físico para aliviar a dor. 
Uma revisão sistemática realizada pelos pesquisadores chineses avaliou outras 23 revisões sistemáticas sobre acupuntura e terapias relacionadas, como a moxabustão e a estimulação elétrica nervosa transcutânea, em relação a sua eficácia e segurança, visto que são frequentemente usadas na China para controlar os sintomas relacionados ao câncer. A qualidade metodológica das 23 revisões foi avaliada pelo Instrumento de Qualidade Metodológica de Revisões Sistemáticas, sendo considerada satisfatória. Após sua avaliação, os autores concluíram que existem evidências para os efeitos terapêuticos da acupuntura no manejo da fadiga, náuseas e vômitos induzidos pela quimioterapia e leucopenia, mas que as evidências em relação ao tratamento da dor relacionada ao câncer, ondas de calor e soluços e melhora da qualidade de vida dos pacientes são conflitantes. Como nenhum efeito adverso grave foi relatado em qualquer estudo, reconhecem que a acupuntura parece ser relativamente segura, podendo ser considerada como uma forma complementar de cuidados paliativos para o câncer (WU X, et al., 2015).

Segundo Lau $\mathrm{CH}$, et al. (2016) realizam estudo semelhante, analisando estudos publicados em cinco bancos de dados internacionais e três chineses, onde concluíram que a acupuntura e as terapias relacionadas são eficazes na redução da dor, fadiga e na melhoria da qualidade de vida quando comparadas à intervenção convencional isolada entre pacientes com câncer. Também indicam que seu uso para o manejo da anorexia, redução da constipação, parestesia e disestesia, insônia e edema de membros em pacientes com câncer necessita de futuros ensaios clínicos randomizados.

Em outro estudo realizado na China, os pesquisadores avaliaram o uso de fitoterápicos no manejo dos sintomas para o tratamento paliativo de câncer, que é muito comum em populações chinesas, em busca de evidências clínicas sobre sua eficácia. Foi conduzida uma revisão sistemática com meta-análise para resumir os resultados de ensaios clínicos controlados randomizados com foco em sintomas que são subtratados em $\mathrm{CP}$ convencionais. Concluíram que a fitoterapia combinada ao tratamento convencional reduziu significativamente dor e constipação, devendo ser considerada como um complemento aos cuidados convencionais (CHUNG VC, et al., 2016).

Axtner J, et al. (2016), realizou um estudo na Alemanha e foi investigada a implementação de uma TC e o tempo de sobrevivência de pacientes que sofrem de câncer pancreático avançado. Foi feita a análise da sobrevida de 240 pacientes empregando modelos de risco proporcional multivariáveis usando diferentes funções de distribuição paramétrica e comparados pacientes que receberam apenas quimioterapia, uma combinação de quimioterapia e tratamento com Viscum album (VA) e somente tratamento VA. Os resultados demonstraram uma excelente aceitação da TC $(93 \%)$ e os pacientes com quimioterapia combinada e terapia de VA apresentaram desempenho significativamente melhor do que os pacientes que receberam apenas quimioterapia (12,1 a 7,3 meses de sobrevida). Ainda, os pacientes que receberam apenas a terapia VA mostraram sobrevida mais longa do que aqueles que não receberam quimioterapia nem terapia VA (5,4 a 2,5 meses). Esse estudo demonstra a importância e o potencial existente para pesquisas em serviços de saúde, mostrando que o tratamento de fitoterapia pode ser implementado com sucesso no cuidado diário de pacientes que sofrem de câncer pancreático avançado.

Grègoire C, et al. (2017) analisaram 3 intervenções psicossociais para reduzir o estresse emocional, dificuldades de sono e fadiga de 123 pacientes com câncer de mama: terapia cognitivo-comportamental (TCC), yoga e hipnose. Os resultados foram comparados com um grupo controle em um acompanhamento de 9 meses. Foi constatada diminuição da ansiedade, depressão e fadiga no grupo de hipnose, e uma diminuição da ansiedade no grupo de yoga, não havendo melhorias significativas para as variáveis investigadas nos grupos TCC e controle. Os resultados mostraram que as intervenções de hipnose e yoga parecem ser uma abordagem interessante para melhorar o bem-estar dos pacientes com câncer de mama.

Em relação ao uso de TC em CP em crianças com câncer, o estudo de pesquisadores dos Estados Unidos da América (EUA) e do Canadá, foi realizou a avaliação de uma variedade de indicações de cuidados de apoio para essa população, por meio de uma revisão sistemática de ensaios clínicos randomizados e controlados. Quarenta e quatro estudos preencheram os critérios de inclusão. Entre eles, 2 ensaios clínicos eram sobre acupuntura, 1 sobre aromaterapia, 9 avaliaram massagem terapêutica e 32 relataram 
suplementos dietéticos. Seu uso estava relacionado com CP para a prevenção ou manejo da mucosite, perda de peso e neutropenia febril. Os autores encontraram resultados encorajadores para essas intervenções, mas consideraram que a maioria dos estudos foi classificada como de baixa a razoável qualidade (RADOSSI AL, et al., 2017),

Shalom S, et al. (2017), em Israel, que procuraram avaliar o impacto das TC no uso das medicações para o tratamento de suporte do câncer, examinando seu impacto na necessidade desses medicamentos. Pacientes com câncer de mama ou ginecológico foram divididos em grupo tratamento e grupo controle. $O$ grupo tratamento recebeu sessões semanais de tratamento com acupuntura e outras modalidades manuais e de movimento (acupressão, reflexologia, método Feldenkrais) e terapias mente-corpo-espírito (imaginação guiada, musicoterapia, cuidado espiritual). Os tratamentos foram adaptados as necessidades de mudança do paciente (toxicidades induzidas pela quimioterapia, preocupações relacionadas à qualidade de vida) e feedback de cuidadores e prestadores de serviços de saúde oncológica. Os resultados demonstraram uma diminuição no uso de analgésicos não opioides em 6 semanas no grupo de tratamento, o que também reduziu o custo dos cuidados de suporte. A análise entre grupos também mostrou uma tendência de redução do uso de ansiolíticos entre as pacientes tratadas.

Segundo Chaol A, et al. (2018), nos EUA, realizaram um estudo randomizado para examinar os efeitos de um programa de yoga tibetano (TYP) versus um programa de alongamento (STP) e cuidados habituais (UC) no sono e fadiga em mulheres com câncer de mama em tratamento quimioterápico. O estudo concluiu que a participação no TYP durante a quimioterapia resultou em benefícios modestos de curto prazo na qualidade do sono, com benefícios a longo prazo surgindo com o tempo para aquelas que praticavam o TYP pelo menos duas vezes por semana.

\section{CONSIDERAÇÕES FINAIS}

Apesar de haver algumas publicações nacionais sobre CP em pacientes oncológicos, esse estudo não encontrou nenhuma que tratasse do tema de terapias complementares, através das bases de dados utilizadas, que foram escolhidas justamente por serem as mais abrangentes. As evidencias do estudo, relatam diversas formas de minimizar o sofrimento dos pacientes oncológico com as terapias vigentes citadas, assim como a alta proporção de aderência desse tipo de terapêutica em diversos países. Dessa forma, torna-se necessário novos estudos científicos, para um melhor conhecimento das especificidades de terapias utilizadas, contribuindo simultaneamente com uma abordagem holística de novas práticas assistenciais.

\section{REFERÊNCIAS}

1. AXTNER $\mathrm{J}$, et al. Health services research of integrative oncology in palliative care of patients with advanced pancreatic câncer. BMC Cancer, 2016; 16(579):1-10.

2. BEN AE, et al. Integrating family medicine and complementary medicine in cancer care: A cross-cultural perspective. Patient Education and Counseling, 2014; 97(1):135-139.

3. BEN AE, et al. Integrative palliative care: complementary medicine in oncology. Clinical Journal of Oncology Nursing, 2017; $21(3):$ 290-293.

4. BRASIL. Ministério da Saúde. Política Nacional de Práticas Integrativas e Complementares no SUS - PNPIC-SUS: atitude de ampliação de acesso. 2ª ed. Brasília: Ministério da Saúde, 2015.

5. CHAOUL A, et al. Randomized trial of Tibetan yoga in patients with breast cancer undergoing chemotherapy. Cancer, $2018 ; 124(1): 36-45$.

6. CHUNG VC, et al. Chinese herbal medicine for symptom management in cancer palliative care: systematic review and meta-analysis. Medicine (Baltimore), 2016; 95(7):1-10.

7. CONRAD AC, et al. Attitudes of members of the German Society for Palliative Medicine toward complementary and alternative medicine for cancer patients. Journal of Cancer Research and Clinical Oncology, 2014; 140(7):1229-1237.

8. GRÉGOIRE C, et al. Group interventions to reduce emotional distress and fatigue in breast cancer patients: a 9-month follow-up pragmatic trial. British Journal of Cancer, 2017;117(10): 1442-1449.

9. INSTITUTO NACIONAL DO CÂNCER (INCA). 2018. Estatísticas do câncer. Publicado em: 26 nov. 2018. Disponível em: <https://www.inca.gov.br/numeros-de-cancer>. Acesso em: 6 mai. 2019. 
10. $\mathrm{LAU} \mathrm{CH}$, et al. Acupuncture and related therapies for symptom management in palliative cancer care: systematic review and meta-analysis. Medicine (Baltimore), 2016; 95(9): 2901.

11. NG CG, et al. The effect of 5 minutes of mindful breathing to the perception of distress and physiological responses in palliative care cancer patients: a randomized controlled study. Journal of Palliative Medicine, 2016; 19 (9): $917-924$.

12. RADOSSI AL, et al. A systematic review of integrative clinical trials for supportive care in pediatric oncology: a report from the International. Supportive Care in Cancer, 2017; 26(2): 375-391.

13. SHALOM SI, et al. Impact of a complementary/integrative medicine program on the need for supportive cancer carerelated medications. Supportive Care in Cancer, 2017; 25(10): 3181-3190.

14. Shin N; Park J. Effect of auricular acupressure on nausea, vomiting, and retching in patients with colorectal cancer receiving chemotherapy. Korean J Adult Nurs., 2018; 30 (3): 227 -34.

15. Vicent, et al, Chinese Herbal Medicine for Symptom Management in Cancer Palliative Care Systematic, Medicine, 2016; 95: 7.

16. WORLD HEALTH ORGANIZATION (WHO). 2018. Disponível em: <http://www.who.int/news-room/factsheets/detail/palliative-care>. Acesso em: 6 mai. 2019.

17. WU X, et al. Effectiveness of acupuncture and related therapies for palliative care of câncer: overview of systematic reviews. Scientific Reports, 2015; 5: 1-15. 